\title{
CrimRxiv
}

\section{Intimate Partner Violence}

Perpetrated by Men

Seeking Help: The

Explanatory Roles of

Psychological Distress and

Affect Dysregulation

Ariane Audet, Audrey Brassard, Caroline Dugal, Aurelie Claing, Marie-Eve Daspe, Claudia Savard, Marie-France Lafontaine, Katherine Peloquin, Natacha Godbout

Published on: Feb 08, 2022

DOI: $10.21428 / c b 6 a b 371.762807 a 3$

License: Creative Commons Attribution 4.0 International License (CC-BY 4.0). 
